\title{
SOME REMARKS ON THE SPACE OF DIFFERENCES OF SUBLINEAR FUNCTIONS
}

Abstract. Two properties concerning the space of differences of sublinear functions $\mathcal{D}(X)$ for a real Banach space $X$ are proved. First, we show that for a real separable Banach space $(X,\|\cdot\|)$ there exists a countable family of seminorms such that $\mathcal{D}(X)$ becomes a Fréchet space. For $X=\mathbb{R}^{n}$ this construction yields a norm such that $\mathcal{D}\left(\mathbb{R}^{n}\right)$ becomes a Banach space. Furthermore, we show that for a real Banach space with a smooth dual every sublinear Lipschitzian function can be expressed by the Fenchel conjugate of the farthest point mapping to its subdifferential at the origin. This leads to a simple family of sublinear functions which contains an exhaustive family of upper convex approximations for any quasidifferentiable function.

1. Introduction. The class of quasidifferentiable functions introduced by V. F. Demyanov and A. M. Rubinov [2] is of considerable importance in nonsmooth optimization. Let us recall some notations as given in [6]:

Let $(X,\|\cdot\|)$ be a real normed vector space, $X^{\prime}$ its topological dual, and $U \subseteq X$ an open subset of $X$. The dual norm of $X$ will be denoted by $\|\cdot\|^{*}$. Moreover, let $\langle\cdot, \cdot\rangle: X^{\prime} \times X \rightarrow \mathbb{R}$ be the dual pairing given by $\langle v, x\rangle:=v(x)$.

A continuous real-valued function $f: U \rightarrow \mathbb{R}$ is said to be quasidifferentiable at $x_{0} \in U$ if the following two conditions are satisfied:

(a) For every $g \in X \backslash\{0\}$ the directional derivative

$$
\left.\frac{d f}{d g}\right|_{x_{0}}=\lim _{t \rightarrow 0+} \frac{f\left(x_{0}+t g\right)-f\left(x_{0}\right)}{t}
$$

exists.

1991 Mathematics Subject Classification: 26A27, 90C30.

Key words and phrases: quasidifferentiable function, sublinear function, upper convex approximation, Fenchel conjugation. 
(b) There exist two sets $\left.\underline{\partial} f\right|_{x_{0}},\left.\bar{\partial} f\right|_{x_{0}} \in \mathcal{K}\left(X^{\prime}\right)$ such that

$$
\left.\frac{d f}{d g}\right|_{x_{0}}=\max _{\left.v \in \underline{\partial} f\right|_{x_{0}}}\langle v, g\rangle+\min _{\left.w \in \bar{\partial} f\right|_{x_{0}}}\langle w, g\rangle .
$$

Here $\mathcal{K}\left(X^{\prime}\right)$ denotes the collection of all nonempty weak*-compact convex subsets of $X^{\prime}$. We remark that by the Alaoglu theorem (see [11], p. 228) the elements of $\mathcal{K}\left(X^{\prime}\right)$ are bounded in the dual norm.

A real-valued function $p: X \rightarrow \mathbb{R}$ is called sublinear if

(i) $p(t x)=t p(x)$ for all $x \in X$ and $t \in \mathbb{R}_{+}:=\{t \in \mathbb{R} \mid t \geq 0\}$,

(ii) $p(x+y) \leq p(x)+p(y)$ for all $x, y \in X$.

It was shown by L. Hörmander [5] that a sublinear function $p: X \rightarrow \mathbb{R}$ is Lipschitzian if and only if its subdifferential at the origin

$$
\left.\partial p\right|_{0}:=\left\{v \in X^{\prime} \mid\langle v, x\rangle \leq p(x), x \in X\right\}
$$

is an element of $\mathcal{K}\left(X^{\prime}\right)$. Since every Lipschitzian sublinear function $p: X \rightarrow$ $\mathbb{R}$ can be expressed as

$$
p(x)=\max _{a \in A}\langle a, x\rangle
$$

for some set $A \in \mathcal{K}\left(X^{\prime}\right)$, the condition (b) is equivalent to the requirement that the directional derivative as a function of the direction $g$ can be expressed as the difference of two sublinear functions. By

$$
\mathcal{P}(X):=\{p: X \rightarrow \mathbb{R} \mid p \text { is sublinear }\}
$$

we denote the convex cone of all real-valued sublinear functions defined on $X$ and by

$$
\mathcal{D}(X):=\{\varphi=p-q \mid p, q \in \mathcal{P}(X)\}
$$

the real vector space of differences of sublinear functions. This space is a lattice with respect to the pointwise max and min operations (see [2], p. 74).

The representation (1) of sublinear functions yields a representation of $\varphi \in \mathcal{D}(X)$ in terms of a pair of compact convex sets $(A, B) \in \mathcal{K}\left(X^{\prime}\right) \times \mathcal{K}\left(X^{\prime}\right)$. However, this representation is no longer unique. In fact,

$$
\max _{a \in A}\langle a, x\rangle-\max _{b \in B}\langle b, x\rangle=\max _{c \in C}\langle c, x\rangle-\max _{d \in D}\langle d, x\rangle
$$

if and only if $A+D=B+C$, where + denotes the usual Minkowski addition, i.e. $A+B=\left\{x \in X^{\prime} \mid x=a+b, a \in A, b \in B\right\}$. This motivates the introduction of the following equivalence relation $\sim$ on $\mathcal{K}\left(X^{\prime}\right) \times \mathcal{K}\left(X^{\prime}\right)$ :

$$
(A, B) \sim(C, D) \text { if and only if } A+D=B+C .
$$

Some recent work deals with minimal pairs of the corresponding equivalence classes with respect to the natural ordering

$$
(A, B) \leq(C, D) \text { if and only if } A \subseteq C \text { and } B \subseteq D
$$


in locally convex topological vector spaces. It was proved by S. Scholtes and J. Grzybowski that minimal pairs in the two-dimensional space are unique up to translation. This is not true for higher dimensions as shown by a counterexample of J. Grzybowski (see [4], [7]-[9], [13]).

For a real separable Banach space $(X,\|\cdot\|)$ we construct a sequence of pseudonorms such that the space $\mathcal{D}(X)$ becomes complete, i.e. a Fréchet space. In the case where $X=\mathbb{R}^{n}$, all these pseudonorms coincide, and $\mathcal{D}\left(\mathbb{R}^{n}\right)$ becomes a Banach space. The second section finishes by an investigation of the compatibility of this norm with the minimality concept introduced above.

For a real Banach space with a smooth dual we then introduce a simple family of sublinear functions which contains an exhaustive family of upper convex approximations for any quasidifferentiable function. Finally, this result will be expressed in terms of the Fenchel conjugate (see [10], Part III, $\S 12)$ of the farthest point mapping to the subdifferential at the origin.

2. The completeness of $\mathcal{D}\left(\mathbb{R}^{n}\right)$. A result more general than the following theorem is stated in a paper by U. Cegrell [1]. However, so far it has not been used within the context of quasidifferentiable functions. We give an adapted version of the proof.

Theorem 1. Let $(X,\|\cdot\|)$ a real separable Banach space. Then $\mathcal{D}(X)$ is a Fréchet space with the topology defined by the seminorms $\|\cdot\|_{\Delta_{n}}: \mathcal{D}(X) \rightarrow$ $\mathbb{R}_{+}$given by

$$
\|\varphi\|_{\Delta_{n}}=\min \left\{\max \left\{\max _{\substack{\|x\| \leq 1 \\ x \in L_{n}}} p(x), \max _{\substack{\|x\| \leq 1 \\ x \in L_{n}}} q(x)\right\} \mid \varphi=p-q, p, q \text { are sublinear }\right\}
$$

where $\left(L_{n}\right)_{n \in \mathbb{N}}$ is an increasing sequence of finite-dimensional spaces whose union is a dense linear subspace of $X$.

Proof. First, observe that for a real normed vector space $(Z,\|\cdot\|)$ a sublinear function $p: Z \rightarrow \mathbb{R}$ is continuous if and only if there exists $M>0$ such that $|p(h)| \leq M\|h\|$ for all $h \in Z$. This implies that for every dense linear subspace $Y \subseteq Z$ we have $\mathcal{P}(Y)=\mathcal{P}(Z)$.

This fact will now be applied. Since $X$ is separable, there exists a sequence $\left(L_{n}\right)_{n \in \mathbb{N}}$ of finite-dimensional linear subspaces of $X$, where $L_{n} \subset$ $L_{n+1}$ for every $n \in \mathbb{N}$, and where $Y:=\bigcup_{n \in \mathbb{N}} L_{n}$ is a dense linear subspace of $X$. Let $B:=\{x \in Y \mid\|x\| \leq 1\}$ denote the unit ball of $Y$ in the norm induced from $X$. Since $\varphi \in \mathcal{D}(Y)$ is uniquely determined by its values in $B$, the space $\mathcal{D}(Y)$ can be considered as a linear subspace of the real vector space

$$
\mathcal{C}_{0}(B):=\{\psi: B \rightarrow \mathbb{R} \mid \psi \text { is continuous }\}
$$


Now $B=\bigcup_{n \in \mathbb{N}}\left(B \cap L_{n}\right)$ is a countable union of compact sets. It is shown in $[11]$, p. 25 , that the vector space $\mathcal{C}_{0}(B)$ can be endowed with a countable family of seminorms $\|\cdot\|_{\Delta_{n}}: \mathcal{D}(X) \rightarrow \mathbb{R}_{+}$given by

$$
\|\varphi\|_{\Delta_{n}}=\min \left\{\max \left\{\max _{\substack{\|x\| \leq 1 \\ x \in L_{n}}} p(x), \max _{\substack{\|x\| \leq 1 \\ x \in L_{n}}} q(x)\right\} \mid \varphi=p-q, p, q \text { are sublinear }\right\}
$$

such that $\mathcal{C}_{0}(B)$ becomes a Fréchet space.

We now show that $\mathcal{P}(Y)$ is a closed subset of $\mathcal{C}_{0}(B)$. For this observe that for every $x \in B$ the mapping

$$
\delta_{x}: \mathcal{C}_{0}(B) \rightarrow \mathbb{R}, \quad \delta_{x}(\psi)=\psi(x),
$$

is a continuous linear functional. Now we consider only $x, y \in \frac{1}{2} B$ and $0 \leq t \leq 1$ and observe that subadditivity and homogeneity on the unit ball are sufficient to characterize sublinear functions on the whole space. Thus in this notation $\varphi(t x)=t \varphi(x)$ can formally be expressed as $\left(\delta_{t x}-t \delta_{x}\right)(\varphi)=0$, and $\varphi(x+y) \leq \varphi(x)+\varphi(y)$ as $\left(\delta_{x+y}-\delta_{x}-\delta_{y}\right)(\varphi) \leq 0$, for every $x, y \in \frac{1}{2} B$ and $0 \leq t \leq 1$. Since both

$$
H_{t, x}=\left\{\varphi \in \mathcal{C}_{0}(B) \mid\left(\delta_{t x}-t \delta_{x}\right)(\varphi)=0\right\}
$$

and

$$
S_{x, y}=\left\{\varphi \in \mathcal{C}_{0}(B) \mid\left(\delta_{x+y}-\delta_{x}-\delta_{y}\right)(\varphi) \leq 0\right\}
$$

are closed, it follows that

$$
\mathcal{P}\left(\mathbb{R}^{n}\right)=\bigcap_{t \in \mathbb{R}_{+}} \bigcap_{x \in B} H_{t, x} \cap \bigcap_{x, y \in B} S_{x, y}
$$

is also a closed subset of $\mathcal{C}_{0}(B)$. In [12], p. 221, it is proved that if $\left(E,\left(\|\cdot\|_{E, n}\right)\right)$ is a real Fréchet space and $K \subseteq E$ a closed convex cone, then the linear space

$$
\Delta K=\{z=u-v \mid u, v \in K\}
$$

endowed with the family of seminorms

$$
\|z\|_{K, n}=\inf \left\{\max \left\{\|u\|_{E, n},\|v\|_{E, n}\right\} \mid z=u-v\right\}
$$

is a Fréchet space as well. This completes the proof.

Since in the finite-dimensional case the unit ball $B$ is compact, the same proof yields:

Corollary 1. Let $\|\cdot\|: \mathbb{R}^{n} \rightarrow \mathbb{R}_{+}$be a norm on $\mathbb{R}^{n}$. Then $\mathcal{D}\left(\mathbb{R}^{n}\right)$ endowed with the norm $\|\cdot\|_{\Delta}: \mathcal{D}\left(\mathbb{R}^{n}\right) \rightarrow \mathbb{R}_{+}$given by

$$
\|\varphi\|_{\Delta}=\min \left\{\max \left\{\max _{\|x\| \leq 1} p(x), \max _{\|x\| \leq 1} q(x)\right\} \mid \varphi=p-q, p, q \text { are sublinear }\right\}
$$

is a Banach space. 
Note that on $\mathcal{P}\left(\mathbb{R}^{n}\right)$ the norm $\|\cdot\|_{\Delta}$ coincides with the supremum norm. This norm can also be expressed as

(3)

$$
\begin{aligned}
& \|\varphi\|_{\Delta} \\
& \quad=\min \left\{\max \left\{\max _{a \in A}\|a\|^{*}, \max _{b \in B}\|b\|^{*}\right\} \mid \varphi(x)=\max _{a \in A}\langle a, x\rangle-\max _{b \in B}\langle b, x\rangle\right\},
\end{aligned}
$$

where $\|a\|^{*}=\max _{\|x\| \leq 1}\langle a, x\rangle$ denotes the corresponding dual norm. One might hope that the pair $(A, B) \in \mathcal{K}\left(\mathbb{R}^{n}\right) \times \mathcal{K}\left(\mathbb{R}^{n}\right)$ for which the norm (3) is attained is minimal in the sense of $(2)$. This, however, is not true in general as the following example shows:

ExAmple 1. Let $\|\cdot\|=\|\cdot\|^{*}=\|\cdot\|_{2}$ be the Euclidean norm, and

$$
\begin{gathered}
A=\operatorname{conv}\left\{\left[\begin{array}{l}
2 \\
1
\end{array}\right],\left[\begin{array}{l}
1 \\
2
\end{array}\right]\right\}, \quad B=\operatorname{conv}\left\{\left[\begin{array}{l}
-2 \\
-1
\end{array}\right],\left[\begin{array}{l}
-1 \\
-1
\end{array}\right],\left[\begin{array}{l}
-1 \\
-2
\end{array}\right]\right\}, \\
C=\operatorname{conv}\left(A \cup\left\{\left[\begin{array}{l}
1.5-\varepsilon \\
1.5-\varepsilon
\end{array}\right]\right\}\right), \quad D=\operatorname{conv}\left(B \cup\left\{\left[\begin{array}{l}
-1.5-\varepsilon \\
-1.5-\varepsilon
\end{array}\right]\right\}\right),
\end{gathered}
$$

where conv denotes the convex hull operator and $\varepsilon>0$. The pair $(A, B)$ is minimal, $(C, D)$ is an equivalent non-minimal pair, but for $\varepsilon \leq(\sqrt{10}-3) / 2$ the minimum in (3) is attained for both pairs.

3. An exhaustive family of upper convex approximations. Let $(X,\|\cdot\|)$ be a real normed vector space, $U \subseteq X$ an open subset of $X$, and $X^{\prime}$ the topological dual endowed with the dual norm $\|\cdot\|^{*}$. Moreover, let $f: U \rightarrow \mathbb{R}$ be directionally differentiable at $x_{0} \in U$. If $p \in \mathcal{P}(X)$ satisfies

$$
p(g) \geq\left.\frac{d f}{d g}\right|_{x_{0}} \quad \text { for all } g \in X,
$$

then $p$ is called an upper convex approximation of $f$ at $x_{0}$. A family $\Phi_{f, x_{0}} \subseteq$ $\mathcal{P}(X)$ of upper convex approximations of $f$ at $x_{0}$ which satisfies

$$
\inf _{p \in \Phi_{f, x_{0}}} p(g)=\left.\frac{d f}{d g}\right|_{x_{0}}
$$

is called an exhaustive family of upper convex approximations of $f$ at $x_{0}$ (see $[2])$.

If $f$ is quasidifferentiable at $x_{0}$, an obvious exhaustive family of upper convex approximations is given by

$$
\widehat{\Phi}_{f, x_{0}}=\left\{p \in \mathcal{P}(X)\left|p(g)=\max _{\left.v \in \underline{\partial} f\right|_{x_{0}}}\langle v, g\rangle+\langle w, g\rangle, w \in \bar{\partial} f\right|_{x_{0}}\right\} .
$$

Note that $\widehat{\Phi}_{f, x_{0}}$ may contain any sublinear function, and thus the union of all possible $\Phi_{f, x_{0}}$ is infinite-dimensional even if $X$ is finite-dimensional. We want to show that it is sufficient to choose $\Phi_{f, x_{0}}$ from a smaller subset $\Phi \subseteq \mathcal{P}(X)$ independent of $f$ and $x_{0}$ which is finite-dimensional for $X=\mathbb{R}^{n}$. 
From (4) it follows that this requires another representation of sublinear functions.

THEOREM 2. Let $(X,\|\cdot\|)$ be a real Banach space such that the topological dual $X^{\prime}$ endowed with the dual norm $\|\cdot\|^{*}$ is smooth. For $p \in \mathcal{P}(X)$ define

$$
\Lambda_{p}=\left\{(\kappa, l) \in \mathbb{R}_{+} \times X^{\prime} \mid \kappa=\sup _{\left.v \in \partial p\right|_{0}}\|l+v\|^{*}\right\} .
$$

Then, for all $x \in X$,

$$
p(x)=\inf _{(\kappa, l) \in \Lambda_{p}}\{\kappa\|x\|-\langle l, x\rangle\}
$$

Proof. Since for $x=0$ the identity (5) is trivial, we may assume that $x \in X \backslash\{0\}$. It follows from the definition of the dual norm that

$$
\begin{aligned}
\inf _{(\kappa, l) \in \Lambda_{p}}\{\kappa\|x\|-\langle l, x\rangle\} & =\inf _{l \in X^{\prime}} \sup _{\left.v \in \partial p\right|_{0}}\left\{\|l+v\|^{*}\|x\|-\langle l, x\rangle\right\} \\
& \geq \inf _{l \in X^{\prime}} \sup _{\left.v \in \partial p\right|_{0}}\{\langle l+v, x\rangle-\langle l, x\rangle\} \\
& =\sup _{\left.v \in \partial p\right|_{0}}\langle v, x\rangle=p(x) .
\end{aligned}
$$

To prove the reverse inequality, we will show that for every $x_{0} \in X \backslash\{0\}$ and every $\varepsilon>0$ there exists a $\left(\kappa^{\varepsilon}, l^{\varepsilon}\right) \in \Lambda_{p}$ such that

$$
\kappa^{\varepsilon}\left\|x_{0}\right\|-\left\langle l^{\varepsilon}, x_{0}\right\rangle \leq p\left(x_{0}\right)+\varepsilon .
$$

A well-known corollary of the Hahn-Banach theorem states that in any normed linear space $X, \max _{\|w\|^{*}=1}\left\langle w, x_{0}\right\rangle$ is attained for some $w_{0} \in X^{\prime}$. This implies that, for every $v \in X^{\prime}$,

$$
\begin{aligned}
\left\|w_{0}+v\right\|^{*} & =\sup _{\|x\|=1}\left\langle w_{0}+v, x\right\rangle \geq\left\langle w_{0}, x_{0} /\left\|x_{0}\right\|\right\rangle+\left\langle v, x_{0} /\left\|x_{0}\right\|\right\rangle \\
& =\left\|w_{0}\right\|^{*}+\left\langle v, x_{0} /\left\|x_{0}\right\|\right\rangle
\end{aligned}
$$

and therefore $x_{0} /\left.\left\|x_{0}\right\| \in \partial\|\cdot\|^{*}\right|_{w_{0}}$. From the assumption that $\|\cdot\|^{*}$ is differentiable, it follows that $\left.\partial\|\cdot\|^{*}\right|_{w_{0}}$ has exactly one element, and thus we obtain

$$
\left\|w_{0}+\mu v\right\|^{*}=\left\|w_{0}\right\|^{*}+\mu\left\langle v, x_{0} /\left\|x_{0}\right\|\right\rangle+o(\mu),
$$

where $\mu \in \mathbb{R}$ and $\lim _{\mu \rightarrow 0} o(\mu) / \mu=0$. Choosing $l^{\varepsilon}=w_{0} / \mu$, we therefore get

$$
\begin{aligned}
\kappa^{\varepsilon}\left\|x_{0}\right\|-\left\langle l^{\varepsilon}, x_{0}\right\rangle & =\sup _{\left.v \in \partial p\right|_{0}}\left\{\left\|\mu^{-1} w_{0}+v\right\|^{*}\left\|x_{0}\right\|-\mu^{-1}\left\langle w_{0}, x_{0}\right\rangle\right\} \\
& =\sup _{\left.v \in \partial p\right|_{0}}\left\{\mu^{-1}\left\|x_{0}\right\|\left(\left\|w_{0}+\mu v\right\|^{*}-1\right)\right\} \\
& =\sup _{\left.v \in \partial p\right|_{0}}\left\{\mu^{-1}\left\|x_{0}\right\|\left(\left\|w_{0}\right\|^{*}+\mu\left\langle v, x_{0} /\left\|x_{0}\right\|\right\rangle+o(\mu)-1\right)\right\} \\
& =\sup _{\left.v \in \partial p\right|_{0}}\left\{\left\langle v, x_{0}\right\rangle+o(\mu) / \mu\right\}=p\left(x_{0}\right)+o(\mu) / \mu .
\end{aligned}
$$


Hence the inequality (6) is satisfied for $\mu$ small enough. This completes the proof.

Note that Theorem 2 does not hold for arbitrary norms as the following example shows:

EXAMPle 2. Let $p \in \mathcal{P}\left(\mathbb{R}^{2}\right)$ be defined as $p(x)=\max \left\{x_{1}, x_{2}\right\}$. Then for any $\Lambda_{p} \subseteq \mathbb{R}_{+} \times \mathbb{R}^{2}$ it is not possible to express $p$ as

$$
p(x)=\inf _{(\kappa, l) \in \Lambda_{p}}\left\{\kappa\|x\|_{1}-\langle l, x\rangle\right\},
$$

since taking $x \in\{(1,0),(0,1),(1,1)\}$, we obtain

$$
\begin{aligned}
\kappa-l_{1} \geq 1 & \text { for all }(\kappa, l) \in \Lambda_{p}, \\
\kappa-l_{2} \geq 1 & \text { for all }(\kappa, l) \in \Lambda_{p}, \\
2 \kappa^{\varepsilon}-l_{1}^{\varepsilon}-l_{2}^{\varepsilon} \leq 1+\varepsilon & \text { for all } \varepsilon>0 \text { and some }\left(\kappa^{\varepsilon}, l^{\varepsilon}\right) \in \Lambda_{p},
\end{aligned}
$$

respectively. Summing up the first two inequalities yields the required contradiction with the third inequality.

Theorem 2 can also be stated in a different way:

Theorem $2^{\prime}$. Let $(X,\|\cdot\|)$ be a real Banach space with $\left(X^{\prime},\|\cdot\|^{*}\right)$ smooth. Let $p \in \mathcal{P}(X)$ and let

$$
\Pi_{\left.\partial p\right|_{0}}: X^{\prime} \rightarrow \mathbb{R} \quad \text { with } \quad \Pi_{\left.\partial p\right|_{0}}(l):=\sup _{\left.v \in \partial p\right|_{0}}\|l+v\|^{*}
$$

be the farthest point mapping with respect to the subdifferential of $p$ at the origin. Then, for all $x \in X$,

$$
p(x)=-\|x\| \Pi_{\left.\partial p\right|_{0}}^{*}(x /\|x\|),
$$

where $\Pi_{\left.\partial p\right|_{0}}^{*}$ denotes the Fenchel conjugate of $\Pi_{\left.\partial p\right|_{0}}$.

Proof. We have

$$
\begin{aligned}
p(x) & =\inf _{l \in X^{\prime}}\left\{\Pi_{\left.\partial p\right|_{0}}(l)\|x\|-\langle l, x\rangle\right\}=-\|x\| \sup _{l \in X^{\prime}}\left\{\langle l, x /\|x\|\rangle-\Pi_{\left.\partial p\right|_{0}}(l)\right\} \\
& =-\|x\| \Pi_{\left.\partial p\right|_{0}}^{*}(x /\|x\|) . \text { - }
\end{aligned}
$$

Corollary 2. Let $(X,\|\cdot\|)$ be a real Banach space with $\left(X^{\prime},\|\cdot\|^{*}\right)$ smooth. Furthermore, let $U \subseteq X$ be an open set and $f: U \rightarrow \mathbb{R}$ be quasidifferentiable at $x_{0} \in U$. Then the family

$$
\Phi=\left\{p \in \mathcal{P}(X) \mid p(g)=\kappa\|g\|-\langle l, g\rangle, \kappa \in \mathbb{R}_{+}, l \in X^{\prime}\right\}
$$

contains an exhaustive family of upper convex approximations of $f$ at $x_{0}$.

Proof. This follows immediately by taking the exhaustive family (4) and expressing the sublinear function $\max _{v \in \underline{\partial f} \mid x_{0}}\langle v, g\rangle$ in terms of (5).

If $X=\mathbb{R}^{n}$, then $\Phi$ is $(n+1)$-dimensional. Note that a reflexive Banach space is strictly convex if and only if $\left(X^{\prime},\|\cdot\|^{*}\right)$ is smooth (see J. Diestel 
[3], p. 24). Hence in this case the condition of smoothness of $\|\cdot\|^{*}$ can be replaced by the condition of strict convexity of $\|\cdot\|$.

Acknowledgements. The authors are thankful to Professors V. F. Demyanov and S. Rolewicz for their useful remarks.

\section{References}

[1] U. Cegrell, On the space of delta-convex functions and its dual, Bull. Math. Soc. Sci. Math. R. S. Roumanie 22 (1978), 133-139.

[2] V. F. Demyanov and A. M. Rubinov, Quasidifferential Calculus, Optimization Software Inc., Publications Division, New York, 1986.

[3] J. Diestel, Geometry of Banach Spaces-Selected Topics, Lecture Notes in Math. 485, Springer, Heidelberg, 1975.

[4] J. Grzybowski, Minimal pairs of compact convex sets, Arch. Math. (Basel), submitted.

[5] L. Hörmander, Sur la fonction d'appui des ensembles convexes dans un espace localement convexe, Ark. Mat. 3 (1954), 181-186.

[6] D. Pallaschke, P. Recht and R. Urbański, On locally Lipschitz quasidifferentiable functions in Banach spaces, Optimization 17 (1986), 287-295.

[7] D. Pallaschke, S. Scholtes and R. Urbański, On minimal pairs of compact convex sets, Bull. Polish Acad. Sci. Math. 39 (1991), 1-5.

[8] D. Pallaschke and R. Urbański, Some criteria for the minimality of pairs of compact convex sets, Z. Oper. Res. 37 (1993), 129-150.

[9] -, 一, Reduction of quasidifferentials and minimal representations, Math. Programming Ser. A, to appear.

[10] R. T. Rockafellar, Convex Analysis, Princeton University Press, Princeton, N.J., 1972 .

[11] S. Rolewicz, Metric Linear Spaces, PWN, Warszawa, and Reidel, Boston, 1984.

[12] H. H. Schäfer, Topological Vector Spaces, Springer, New York, 1971.

[13] S. Scholtes, Minimal pairs of convex bodies in two dimensions, Mathematika 39 (1992), 267-273.

SVEN G. BARTELS AND DIETHARD PALLASCHKE

INSTITUT FÜR STATISTIK UND MATHEMATISCHE WIRTSCHAFTSTHEORIE

UNIVERSITÄT KARLSRUHE

KAISERSTR. 12

D-76128 KARLSRUHE, GERMANY 